\title{
El papel de la discriminación de estímulos en la lealtad hacia un producto de consumo masivo*
}

The Role of Discrimination of Stimuli in the Loyalty Towards a Product of Massive Consumption O papel da discriminação de estímulos na lealdade a um produto de consumo massivo

\author{
Sandra Tobón ${ }^{\star \star}$ \\ Andrés M. Pérez Acosta ${ }^{\star \star}$
}

Fecha de recibido: 16 de octubre de 2015

Fecha de aprobado: 27 de enero de 2016

Doi: http://dx.doi.org/10.12804/rev.univ.empresa. 31.2016 .04

Para citar: Tobón, S., \& Pérez Acosta, A.M. (2016). El papel de la discriminación de estímulos en la lealtad hacía un producto de consumo masivo. Universidad \& Empresa 18(31), 85-101. Doi: http://dx.doi.org/10.12804/ rev.univ.empresa.31.2016.04

\section{RESUMEN}

La lealtad de marca se define como la compra sistemática de una misma marca en distintas instancias de compra y la discriminación de estímulos como la capacidad de los individuos de diferenciar un estímulo de un conjunto con características similares. Esta investigación evalúa el papel de la discriminación de marca/producto por parte de consumidores con historias diferenciales de consumo de un producto de consumo masivo respecto de las distintas

* Este artículo es resultado de la investigación: "Lealtad conductual con discriminación marca/producto entre consumidores con historia diferencial de consumo" que se adelantó para la Maestría en Psicología en la Universidad Nacional de Colombia. El objetivo general de la investigación fue: Evaluar empíricamente el papel de la discriminación de estímulos en la constitución de un concepto de lealtad conductual de marca.

** Magíster en Psicología por Universidad Nacional de Colombia. Coordinadora programa de Administración de Empresas, Corporación Universitaria de Colombia IDEAS, Bogotá. Email: maribeltobon@gmail.com CP: 111321

**** Doctor en Psicología por Universidad de Sevilla (España). Profesor Titular, Programa de Psicología, Universidad del Rosario, Bogotá, Colombia. Email: andres.perez@urosario.edu.co 
opciones en una categoría. Los resultados permiten concluir que la discriminación no es una condición necesaria para la lealtad de marca, el hecho de que el consumidor pueda diferenciar una marca, ya sea por los atributos del producto o las características simbólicas de la marca, no garantiza la compra o consumo exclusiva de la misma. Los resultados se analizaron a la luz de las teorías del mercadeo y psicológicas del consumo y sus implicaciones, en ambas áreas del conocimiento.

Palabras clave: aprendizaje de marca, atributos de producto, discriminación de marca, lealtad de marca.

\section{ABSTRACT}

Brand loyalty is defined as the systemic purchase of a same Brand in different instances of purchase and the discrimination of stimulates as a capability of individuals to differentiate a stimulus from a group with similar characteristics. This research evaluates the role of discrimination of brands/products from consumers with differential histories of consumption of a product of massive consumption according to the different options of a category. The results allow concluding that discrimination is not a condition needed to Brand Loyalty, in this ordering of ideas the fact that the consumer can differentiate a brand, this being for the attributes of the product or the symbolic characteristics of the brand, does not guarantee the purchase or exclusive consumption of the same. The results were analyzed to the light of the marketing and psychological theories of consumptions and its implications in both fields of knowledge.

Keywords: Brand learning, product features, brand discrimination, brand loyalty.

\section{RESUMO}

A lealdade de marca define-se como a compra sistemática de uma mesma marca em distintas instâncias de compra e a discriminação de estímulos como a capacidade dos indivíduos de diferenciar um estímulo de um conjunto de características similares. Esta pesquisa avalia o papel da discriminação de marca/produto por parte de consumidores com histórias diferenciais de consumo de um produto de consumo massivo respeito das distintas opções em uma categoria. Os resultados permitem concluir que a discriminação não é uma condição necessária para a lealdade de marca, de forma que o facto de que o consumidor possa diferenciar uma marca, seja pelos atributos do produto, seja pelas características simbólicas da marca, não garante a compra ou consumo exclusiva da mesma. Os resultados se analisaram à luz das teorias de marketing e psicológicas do consumo e as suas implicações, em ambas as áreas do conhecimento.

Palavras-chave: aprendizagem de marca, atributos de produto, discriminação de marca, lealdade de marca. 


\section{INTRODUCCIÓN}

Lealtad de marca es uno de los conceptos más estudiados en la literatura del marketing y el principal objetivo a alcanzar por los empresarios (Colmenares \& Saavedra, 2007). En su búsqueda, las empresas invierten recursos financieros y humanos para diferenciar sus productos o servicios de la oferta de la competencia, sin embargo, poco interés se ha aplicado a estudiar; tanto a nivel teórico, como práctico, la capacidad que tienen los consumidores de percibiresas diferencias entre las distintas ofertas, es decir sobre la capacidad de discriminación de marca/producto y su papel en la lealtad de marca.

Esta investigación se propone llenar este vacío al evaluar empíricamente la capacidad de discriminación de los consumidores de un producto de consumo masivo de su marca tradicionalmente elegida y evaluar su papel en la lealtad conductual de marca.

El documento está organizado de la siguiente manera: inicialmente se presenta una revisión de la literatura desde el marketing sobre lealtad de marca y las estrategias de diferenciación de productos y luego se revisa los modelos, desde la psicología, de la discriminación de estímulos, para establecer las diferencias entre lealtad de marca con discriminación de estímulos de la recompra por inercia conductual o sin discriminación de marca/producto. Luego se presentan las hipótesis, el método y los resultados encontrados en la investigación empírica que se realizó para poner a prueba las hipótesis que se derivaron de la revisión de la literatura.

\section{REVISIÓN DE LA LITERATURA}

\subsection{Lealtad de marca}

La literatura sobre lealtad de marca se ha clasificado en dos corrientes principales: la primera, la define como una actitud y la segunda como una conducta (Rundle-Thiele, 2005).

La lealtad como actitud es entendida como "la predisposición de un consumidor hacia una marca particular, la cual es una función de un proceso psicológico" (Rundle-Thiele, 2005, p. 31); según esta perspectiva, los consumidores realizan un proceso de análisis de información donde comparan las distintas marcas y atributos de productos de las diversas ofertas disponibles en una categoría de producto. Según dicho análisis se crearía la preferencia hacia una marca particular que explicaría la posterior compra y recompra de la misma; de tal forma, que la preferencia actitudinal sería un antecedente y predictor de la conducta de compra (Kraus, 1995; Sample \& Warland, 1973). 
Por otra parte, la lealtad definida como conducta o lealtad conductual, indica la probabilidad de recompra de una marca en las distintas instancias de elección. Su medición se basada en la historia de compra y consumo de la misma marca, la cual es observable, más no inferida (Bhattacharya, 1997; Chaudhuri \& Holbrook, 2001; Dekimpe, Steenkamp, Mellens \& Vanden, 1997). Tiene la ventaja de no ser incidental, sino de representar una conducta extendida en el tiempo. Esta última es la perspectiva que adoptaremos en la presente investigación.

\subsection{Diferenciación de productos}

La estrategia de diferenciar los productos, como mecanismo para competir en un mercado, se realiza a través del "diseño o la imagen de marca, la tecnología, las características, el servicio al cliente, las redes de distribución u otras" (Porter, 2004, p. 54), siendo la imagen de marca y las características del producto a las que más recursos financieros le dedican las empresas (Chen, 2014).

Las estrategias de diferenciación pueden ser con base en las características del producto (color, tamaño del empaque, etiqueta o sabor) o con base en la calidad del producto, entendida esta última como el conjunto de características que son cruciales para el cliente y que el percibe que a mayor presencia de dicha característica mayor calidad del producto y por la que podría estar dispuesto a pagar más (Vandenbosch \& Weinberg, 1995).

Bajo la premisa de "diferenciarse o desaparecer"(Trout\& Rivkin, 2000), el marketing ha enfatizado en construcción de identidades de marca o producto, de forma que cada marca "sea un sustituto imperfecto con otras marcas para que los compradores sean leales" (Caves \& Williamson, 1985, p. 115); sin embargo, se ha evidenciado cómo la diferenciación que suponen las empresas, en algunos casos no son percibidas por los consumidores o simplemente no parece ser fundamental a la hora de elegir sus productos, ya que, en los casos en los que manifiestan percibir diferencias entre las distintas alternativas no se constituyen en factores modificadores de sus conductas de compra (Romaniuk, Sharp, \& Ehrenberg, 2007).

Lo anterior lleva a que nos preguntemos por el proceso de discriminación de marca/producto, por el que pasa un consumidor para intentar entender su importancia a la hora de comprar o recomprar una marca.

\subsection{Discriminación de marca}

La discriminación de estímulos es una capacidad adaptiva del ser 
humano, resultado del proceso de aprendizaje e interacción con los estímulos ambientales (Dinsmoor, 1995a), por lo tanto, es la forma como los individuos ajustan sus repertorios conductuales, según los contextos, anticipando apropiadamente resultados favorables o desfavorables.

En el ámbito del consumo, el proceso mediante el cual un consumidor discrimina entre las distintas ofertas disponibles en una misma categoría de producto es un fenómeno complejo en el que están implicadas las características físicas de cada producto y marca, así como la historia particular de consumo o compra del usuario con este (Wasserman, Yung, $\&$ Cook, 2004). Mientras un producto y su marca logre ser discriminado por los consumidores de las demás marcas en la categoría, mayores probabilidades tiene el producto de incrementar sus ventas y continuar en el mercado; por su parte, un menor grado de discriminación de la misma (generalización), conlleva menores probabilidades del producto de permanecer en un mercado con altos niveles de competencia. En términos del consumidor, la elección correcta, es decir el producto que mejor satisfaga sus necesidades o deseos, implicará menores costos económicos y de elección en el intercambio.
Una rama de la psicología conductual aplicada, ha estudiado el proceso, a través del cual se construyen en el consumidor el aprendizaje de marca, el cual han denominado como aprendizaje asociativo de marca (Froufe \& Sierra, 1998). Siendo dicho aprendizaje el resultado de una presentación pareada y sistemática de una marca (estímulo condicionado, EC) con un estímulo incondicionado (EI) que puede ser una imagen, sonido, artista u otro estímulo con una alta capacidad de desencadenamiento emotivo, lo que resulta en que la primera EC (marca), adquiere la capacidad de desencadenar la respuesta emotiva sin la presencia del EI (Cruz \& Pérez-Acosta, 2002; De Hower, Baeyens \& Field, 2005; De Houwer, Thomas \& Baeyens, 2001; Froufe \& Sierra, 1998; Gorn, 1982).

Según este modelo del aprendizaje asociativo, la presentación pareada de una marca con un tipo de estímulo con valencia positiva, vía presentación publicitaria, sería suficiente para que los participantes aprendieran a discriminar esa marca particular de las otras en la categoría, sin necesidad de intervenir los atributos del producto.

Por su parte, el modelo del aprendizaje adaptativo, plantea que el aprendizaje es el resultado de la interacción entre estímulos o señales 
(marcas) que son contingentes a la aparición de unos atributos específicos del producto; en la medida en que una marca prediga apropiadamente dichos atributos, mayores probabilidades tendría de ser elegida (Van Osselaer \& Janiszewski, 2001). Según este modelo teórico, para que el aprendizaje tenga lugar, no es suficiente la presentación, con una relación de contigüidad entre el EC y EI, sino que es necesario que exista covariación entre los dos estímulos. El modelo, conocido como el modelo Rescorla \& Wagner, introdujo al modelo estímulo-respuesta de Pavlov, las expectativas de los sujetos experimentales; según este, el aprendizaje es el resultado de la evaluación de los resultados obtenidos de una respuesta particular y lo que el sujeto esperaba obtener.

En el contexto del consumidor Janiszewski \& Van Osselaer (2000) demostraron, a través de una serie de experimentos en los que les dieron a degustar a los participantes ocho muestras de tortas, cada una con una medida distinta de calidad del sabor, la cual dependía del tipo de ingrediente principal que contenía cada producto, que los participantes aprendieron a predecir que producto sabía mejor con base en la asociación previa de marca de ingrediente-sabor.
Según este modelo, la discriminación sería el resultado de un aprendizaje marca-atributos de producto $\mathrm{y}$, en la medida en que la marca prediga mejor los resultados o beneficios que espera obtener un consumidor de un producto, mayores probabilidades tendría dicha marca de ser seleccionada en la siguiente instancia de elección.

En síntesis, la discriminación de marca por parte de un consumidor, reflejaría el nivel de aprendizaje obtenido en la interacción (compra, consumo y exposición) con el producto y todo su componente simbólico que lo caracteriza. Este aprendizaje se podría explicar como un aprendizaje de marca (asociativo o adaptativo) en el que determinadas características emotivamente estimulantes pueden dar cuenta de la preferencia y elección sistemática de dicha marca.

\subsection{Lealtad e inercia conductual}

La respuesta conductual, en la que el consumidor compra sistemáticamente la misma marca, pero lo hace como resultado de un efecto del mantenimiento del statu quo o por inercia (Inman \& Zeelenberg, 2002; Samuelson \& Zechauser, 1988), se caracteriza por ser una conducta en la cual el consumidor no diferencia entre la marca o 
producto que habitualmente adquiere de las demás marcas que conforman una categoría específica. Esta conducta puede ser consecuencia de "una decisión racionalen presencia de costos de transacción y/o incertidumbre; una mala percepción cognitiva o un compromiso psicológico proveniente de costos hundidos [pago ya realizado en una transacción, no reembolsable, que impide que se cambie la decisión tomada], evitación del remordimiento, o un actuar por consistencia" (Samuelson, et al., 1988, p. 33).

El concepto de mantenimiento del statu quo se ha estudiado principalmente desde la economía y en especial con las teorías de elección en condiciones de incertidumbre (Schweitzer, 1994). Según Scheweitzer (1994) cuando las condiciones de elección de un primer momento se mantienen (precio, costos de oportunidad, etc.); adicionalmente, cuando los costos de transacción o de cambio en la elección son superiores al beneficio que se puede obtener, la decisión más "racional" que podría tomar un consumidor es seguir la misma línea conductual inicial, por lo tanto cambiar de marca o producto sería económicamente injustificable. Por otra parte, desde un enfoque totalmente opuesto, Tversky \&
Kahneman (1974) demostraron a través de una serie de experimentos, que en condiciones de aversión al riesgo, se pueden tomar decisiones que propenden por la conservación del statu quo para disminuir las pérdidas, incluso en condiciones donde existirían mayores probabilidades de obtener utilidades superiores si se cambia de opción, esto como consecuencia de una mala percepción cognitiva que impide estimar apropiadamente las probabilidades de ocurrencia de un evento.

En el contexto del consumidor, Inman, et al., (2002) en una serie de cuatro experimentos evaluaron el nivel de remordimiento (regret) que podían sentir los consumidores que cambiaban constantemente de marca de los que elegían siempre la misma marca (recompra), encontraron que, cuando los resultados eran negativos, el sentimiento de remordimiento era menor entre quienes cambiaban de marca (switchers), que entre los que recompraban la misma marca. Estos resultados, sugerirían que el actuar conservando el statu quo, en términos de remordimiento, sería menos aconsejable que el cambiar de marca.

En cuanto a la capacidad de discriminar la marca que usualmente se consume de las otras marcas entre 
los que siempre compran la misma marca y los switchers, Knox, et al., (2001) afirman que los primeros tendrían mayor capacidad de discriminación de marca debido a su mayor conocimiento del producto que acostumbran consumir; mientras que los switchers no encontrarían diferencias entre los productos de las distintas marcas y por tanto su conducta estaría determinada, fundamentalmente, por las variaciones en el precio o en las promociones disponibles en el momento en que realiza la compra. Contrario a lo anterior, Kotler, Armstrong, Saunder \& Wong (1996, p. 48) afirman que los consumidores que siempre recompran la misma marca son "procesadores pasivos de información", y por lo tanto, con una limitada capacidad de discriminación.
En síntesis, la inercia conductual se reflejaría en una compra sistemática de una misma marca, pero donde el consumidor no discriminaría entre los productos y marcas que conforman la categoría. En este caso la marca cumpliría una función nominal más que la de un estímulo que le permite al consumidor discriminar el producto de los otros y donde se reflejaría un aprendizaje de marca sin mediación de los atributos físicos del producto.

\section{HIPÓTESIS}

Partiendo de la literatura sobre la lealtad de marca y la conceptualización, desde la psicología, respecto de los mecanismos de aprendizaje de discriminación de estímulos, se propone el siguiente modelo e hipótesis:

Figura 1. Modelo propuesto

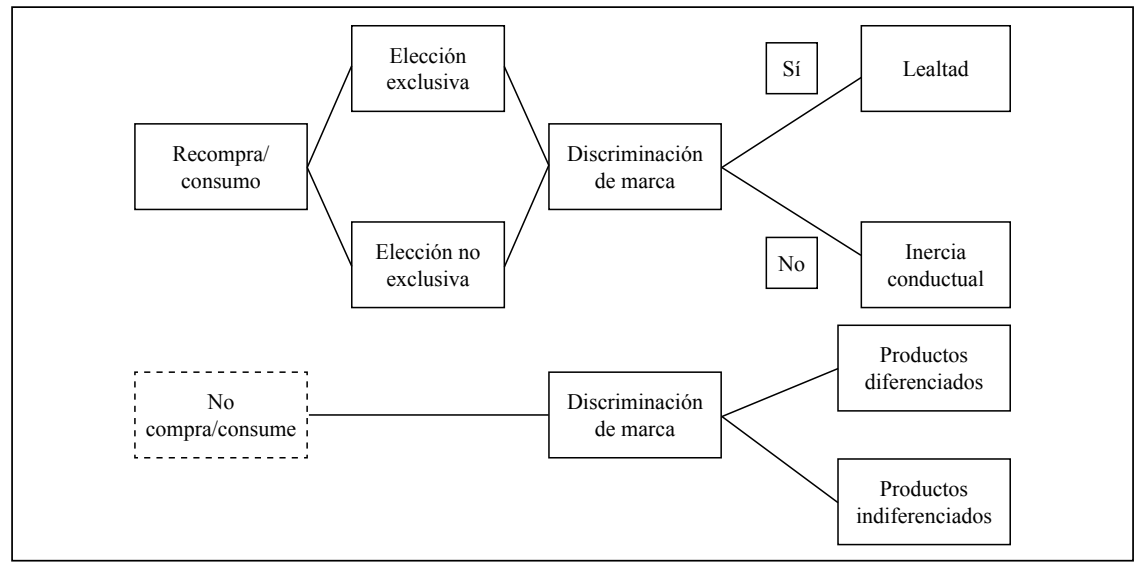

Fuente: elaboración propia. 
H1. Los consumidores que compran distintas marcas (Switchers) presentan menor capacidad de discriminación que los que compran una marca exclusiva en distintas instancias de compra (mayor inercia conductual).

H2. La discriminación de marca, por parte de los consumidores, no es una condición necesaria para la lealtad a la marca.

H3. A mayor frecuencia de consumo del producto, mayor capacidad de discriminación de marca/producto.

\section{METODOLOGÍA}

En procedimiento participaron 31 clientes de un centro comercial de Bogotá D.C. los cuales voluntariamente aceptaron participar firmando el respectivo consentimiento informado.

La elección de los participantes se realizó por conveniencia, según elección y frecuencia de consumo de una o varias marcas de tajada de ponqué cubierta de chocolate durante las compras un fin de semana. Se conformaron tres grupos: el primero (G1) conformado por 12 personas, quienes manifestaron comprar siempre la misma marca del producto, el dos grupo (G2) formado por 10 individuos que compran simultánea o indistintamente dos o más marcas y el tercero (G3) participantes que nunca compran ni consumen el producto.

\subsection{Materiales y estímulos}

Para la prueba de discriminación de marca, se diseñó un aplicativo en Adobe Flash ${ }^{\circledR}$ CS3, denominado Test de discriminación marca, el cual se instaló en un computador portátil Acer ${ }^{\circledR}$. El aplicativo presentaba los estímulos (figura 2) en una secuencia predeterminada, según las especificaciones del procedimiento.

Figura 2. Estímulos utilizados en el estudio

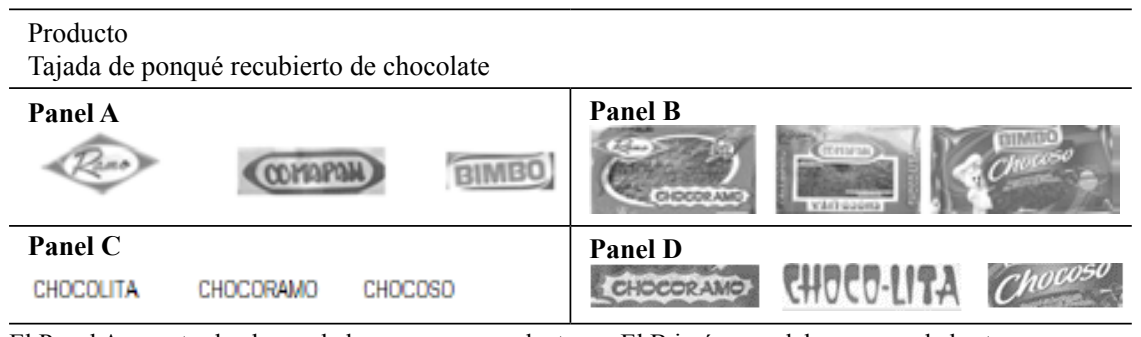

El Panel A muestra los logos de las empresas productoras. El B imágenes del empaque de las tres marcas del producto. El Panel C muestra los nombres de los productos y el Panel D los logo-símbolos.

Fuente: elaboración propia. 
El procedimiento se realizó dentro de las instalaciones de un almacén de cadena, en la ciudad de Bogotá, donde con la debida autorización, se instalaron dos mesas una para la prueba de producto y otra para el computador portátil (discriminación de marca). Estas se ubicaron justo enfrente de la góndola que exhibe los productos de panadería y pasa-bocas.

Para la prueba de producto siguió un procedimiento de doble ciego, donde cada tajada de ponqué de las tres marcas distribuidas en el mercado (Chocolita ${ }^{\circledR}$, Chocoramo ${ }^{\circledR}[s i c]$, Chocoso $\left.{ }^{\circledR}\right)$ se cortó en trozos simétricos de 2.5 por 2.5 centímetros aproximadamente. Cada tipo de producto se depositó en una bandeja desechable de icopor color blanco y se cubrió con otra de las mismas características. Estas muestras de producto se ubicaron de forma contigua y cambiando la posición de derecha a izquierda en cada aplicación, sobre una de las mesas. Adicionalmente, en ella se ubicó los vasos desechables de 3.5 oz y la botella de agua de $1500 \mathrm{ml}$. En la segunda mesa y de forma adyacente se ubicó el computador portátil y una silla.

\subsection{Diseño}

Se realizó un procedimiento no experimental con diseño trasversal Hernández, Fernández-Collado \&
Baptista (2006), donde se evaluó la discriminación a la marca y atributos del producto de un grupo de consumidores organizados en grupos según su reporte de historia de consumo del producto. El procedimiento constó de dos fases: en la primera, se observó la conducta de elección de los clientes en la góndola del supermercado. Con base en ella, se seleccionó los participantes y se les invitó a participar en la siguiente fase. En esta, se evaluó la capacidad de discriminación de los consumidores. Esta fase constó, a su vez, de dos etapas, la etapa de discriminación de marca y la etapa de discriminación de producto.

\section{RESULTADOS Y DISCUSIÓN}

Previo a la realización de los análisis de varianza, de la variable dependiente (tiempo de respuesta), se evaluó el supuesto de normalidad y el supuesto de homogeneidad de varianzas (prueba de Levene) (Ortíz, 2013). El p-valor obtenido en la prueba de Kolmogorov-Smirnov (0.15) y Shapiro-Wilk (0.056) y la prueba de Levene (0.90), nos permite asumir el supuesto de normalidad en la distribución de la variable estudiada, así como nos permite asumir varianzas iguales de las poblaciones de donde se extrajo la muestra en cuanto a la misma variable. 


\subsection{Frecuencia de consumo y compra}

Según el reporte de los participantes, se pudo establecer tres frecuencias de consumo: de 1 a 2 veces por semana (10), de 3 a 4 veces por semana (10), no consumen (10) y (2) que consumen de 1 a 2 veces por mes. Este último grupo se excluyó del análisis por la poca representatividad en la muestra.

\subsection{Discriminación de producto}

En la figura 3 se muestran los resultados de la prueba de discriminación de producto según el grupo al que pertenece el participante. Con esta prueba se evaluó la capacidad de discriminar el producto que usualmente compra y consume el participante de los otros que conforman la oferta disponible teniendo en cuenta las características propias del producto (textura, sabor, forma). Se observa que el número de participantes que discriminaron, en los tres grupos, son mayoritarios a los que no discriminaron. Las diferencias son significativas estadísticamente $(0.0001)$ lo que indica que independientemente de la historia de consumo, los participantes pueden discriminar el producto de su preferencia de las demás alternativas, teniendo en cuenta las características del producto.

Figura 3. Número de participantes que discriminaron en la prueba de producto

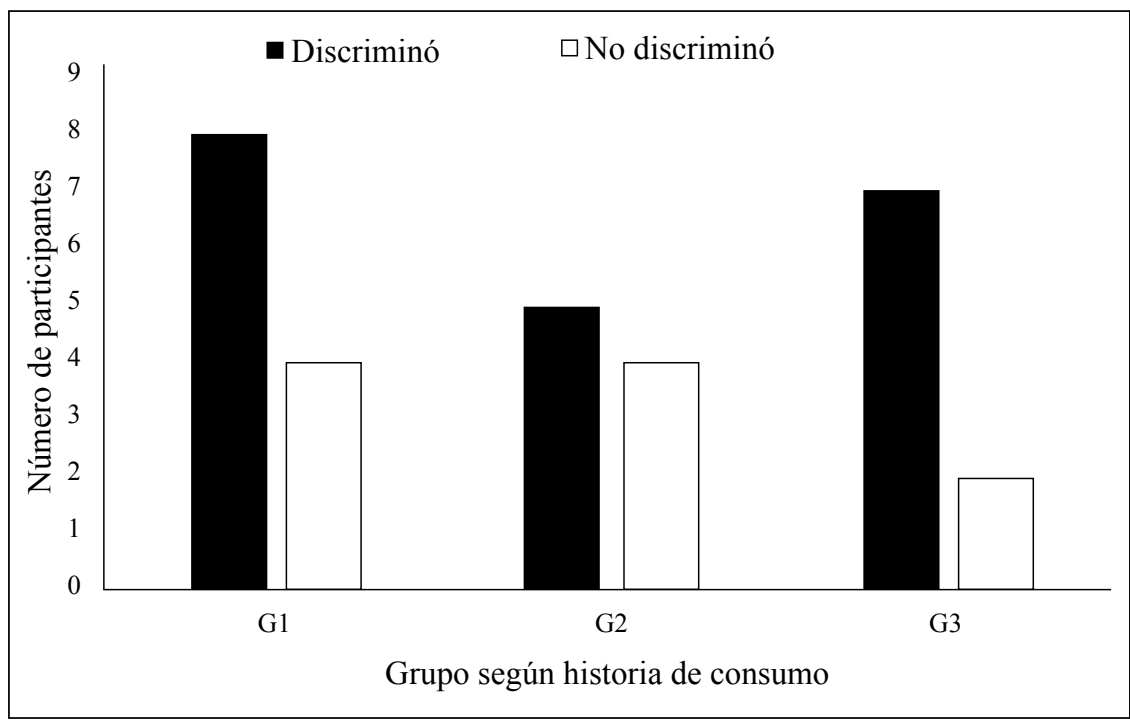

Fuente: elaboración propia. 


\subsection{Discriminación de marca}

En esta prueba se midió la discriminación de marca utilizando el tiempo de respuesta a los estímulos publicitarios que caracterizan cada producto (empaque, nombre del producto, logo del fabricante y logo del producto). La figura 4 muestra la comparación según el tiempo total de respuesta de los tres grupos, no siendo significativo ( $\mathrm{p}>0.001$ ), lo que respalda la hipótesis 1 en el sentido de que el hecho de consumir exclusivamente una marca o variar la elección no implica que se desarrolle mayor capacidad de discriminación de marca.

Figura 4. Tiempo de respuesta (segundos) por grupos

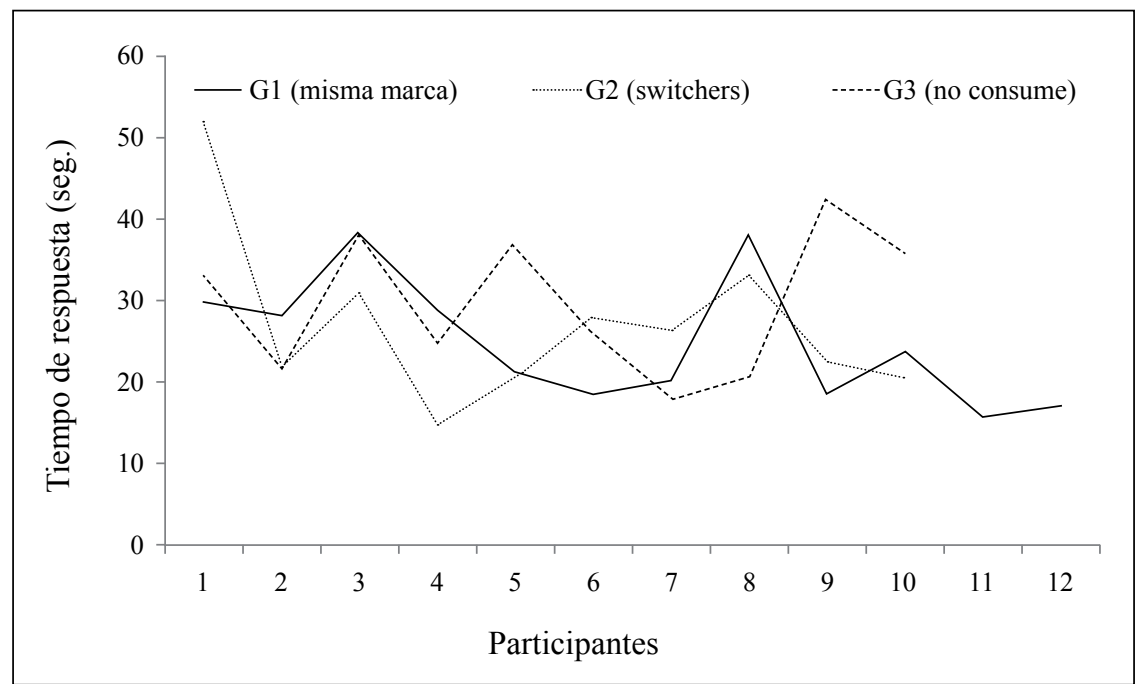

Fuente: elaboración propia.

De lo anterior, se pueden extraer dos conclusiones: primera, el hecho de consumir un mismo producto no implica un conocimiento mayor de los atributos del producto o características de marca como plantean Knox \& Walker (2001) frente a los switchers por lo que no es posible sostener que se presente un aprendizaje progresivo del consumidor hacia su producto que refleje mayores nivel de discriminación (Wasserman, Yung, \& Cook, 2004); segunda, los consumidores habituales no serían "procesadores pasivos de información" como proponen Kotler, et al., (1996), sino que discriminan su producto y marca 
habitual, lo que implica que, para el producto estudiado, el nivel de consumo considerado por inercia es cercano a cero.

Tabla 1. Análisis de varianza (ANOVA) del test de discriminación de marca

\begin{tabular}{|c|c|c|c|}
\hline Tiempo total de prueba & $\mathrm{gl}$ & $\mathrm{F}$ & Sig. \\
\hline \multicolumn{4}{|c|}{ Por grupos } \\
\hline G1-G2-G3 & 2 & 0.826 & 0.448 \\
\hline Intra-grupos & 29 & & \\
\hline Total & 31 & & \\
\hline \multicolumn{4}{|c|}{ Según prueba de producto } \\
\hline Discriminó/No discr. & 1 & 0.025 & 0.874 \\
\hline Intra-grupos & 28 & & \\
\hline Total & 29 & & \\
\hline
\end{tabular}

Fuente: elaboración propia.

En la primera sección de la tabla 1 se presentan los resultados de la comparación de los promedios en el tiempo de respuesta entre los tres grupos (G1, G2 y G3), el p-valor (.448) nos indica que las diferencias no son estadísticamente significativas; por lo tanto, independientemente de la historia de consumo, los participantes lograron discriminar la marca que usualmente consumen de las demás marcas.

La segunda parte de la tabla 1 presenta el resultado de comparar las dos etapas del procedimiento de discriminación (marca y producto), el p-valor (0.874) indica que no existen diferencias significativas en el desempeño de la prueba de discriminación de marca respecto al desempeño en la de discriminación de producto. Es decir, independientemente de que el participante pueda diferenciar o no el producto por sus atributos, logra diferenciar las marcas por su componente simbólico.

Lo anterior sustenta la hipótesis 2; es decir que independiente de la historiade consumo, los participantes pueden discriminar la marca/producto que usualmente consumen; por lo tanto, la discriminación no es una condición necesaria para la lealtad de marca. Estos resultados son coherentes con lo planteado por Foxall, 1999; Foxall, Oliveira-Castro \& Scherezenmaier, (2004) respecto a la diferenciación de las marcas vía actividades de branding, lo que permite 
que los consumidores puedan diferenciar la que usualmente consumen de las otras marcas que conforman la categoría.

A su vez, los resultados sugieren que existen dos tipos de aprendizaje: un aprendizaje de marca y otro de producto implicados en el proceso de elección de marcas/producto tal como plantea Foxall, Oliveira-Castro \& Schrezenmaier (2004), donde dicho aprendizaje es el resultado de la experiencia o historia de consumo o de la exposición sistemática a los mensajes publicitarios (aprendizaje asociativo de marca). A continuación se analiza el papel de la frecuencia de consumo en la discriminación de marca.

La figura 5 relaciona el tiempo de respuesta según la frecuencia de consumo del producto, se encontraron diferencias significativas para los dos grupos $(\mathrm{p}<0.001)$, según la frecuencia de consumo, siendo menor el tiempo de respuesta para los que consumen el producto entre 3-4 por semana.

Figura 5. Tiempo de respuesta según frecuencia de consumo

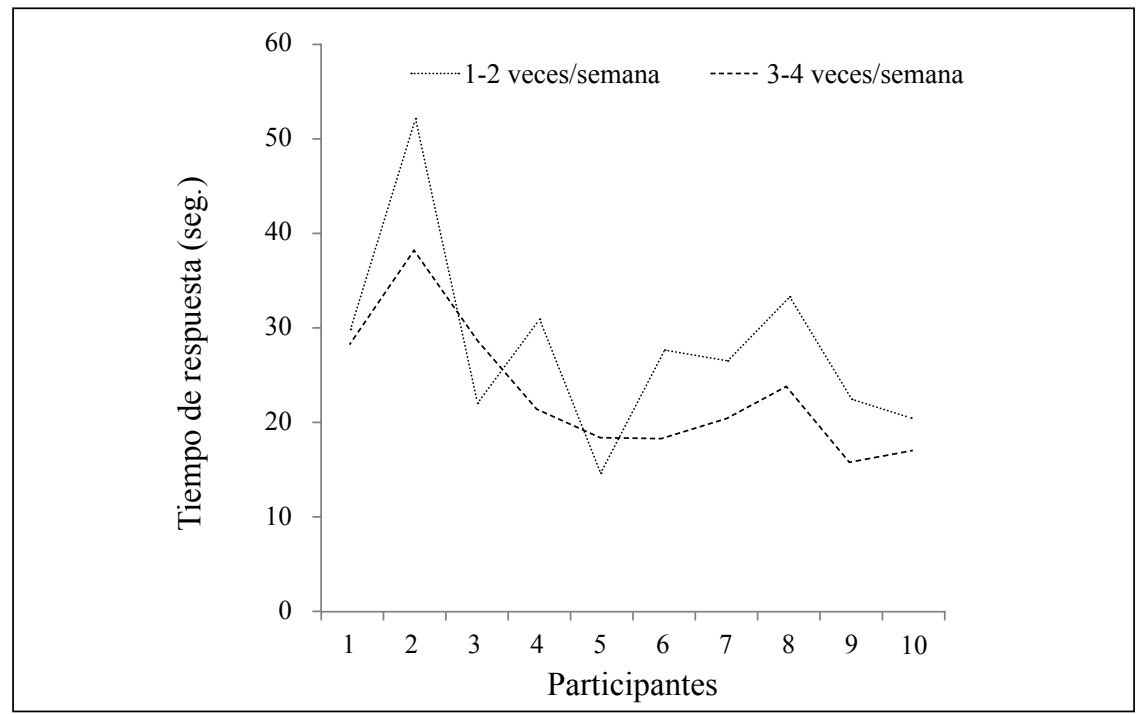

Fuente: elaboración propia.

Lo anterior implica que la Hipótesis 3 estaría sustentada, por lo que sería apropiado afirmar que a mayor frecuencia de consumo del producto, mayor nivel de discriminación del mismo. Estos resultados son cohe- 
rentes con lo que plantean MartínezCarrasco, Brugarolas, Del Campo \& Martínez (2006), en el sentido de que a mayor frecuencia de consumo, mayores probabilidades de discriminar y preferir una marca respecto a las de la competencia. Esto a su vez nos permite afirmar que tanto los atributos del producto, como los mensajes publicitarios son factores que influyen significativamente en la capacidad del consumidor de discriminar la marca.

\section{DISCUSIÓN Y CONCLUSIONES}

Los resultados nos permiten concluir, siguiendo las hipótesis planteadas, que la discriminación de marca, en tanto estímulo, no es una condición necesaria para establecer la lealtad a una marca; de forma que el hecho de que se reconozca la marca o un producto por sus características particulares, no garantiza que el consumidor compre siempre dicha marca. Adicionalmente, la discriminación de la marca por sus componentes simbólicos es independiente de la discriminación por los atributos del producto; de tal forma, que el consumidor obtiene información distinta y relevante, tanto del empaque y diseño publicitario, como de las características físicas del producto. Por último, cuando los productos son altamente diferenciados, ya sea vía branding o caracterización del mismo, independientemente de la frecuencia de consumo, el consumidor puede discriminar su marca predilecta.

\subsection{Implicaciones para la Psicología del Consumidor}

La discriminación de estímulos es un proceso básico de adaptación de los organismos a su ambiente (Dinsmoor, 1995a). La marca y atributos de producto se constituyen en estímulos que señalizan los refuerzos contingentes a la compra de un bien o servicio. La publicidad y demás labores de branding son actividades que permiten diferenciar dos productos funcionalmente equivalentes para facilitar la elección de los consumidores. Por lo tanto, los resultados de la presente investigación permiten establecer cómo los procesos de entrenamiento o sensibilización vía publicitaria y en menor medida el uso o consumo del producto influyen en la capacidad de discriminación de atributos de producto y dimensiones de marca y que esta capacidad no explica el proceso de recompra de un producto.

\subsection{Implicaciones para el Mercadeo}

El pilar fundamental del mercadeo ha sido la diferenciación de marcas 
para la construcción de lealtad de sus clientes. Sin embargo, se ha asumido que los consumidores son pasivos frente a la publicidad y los mensajes a los que son expuestos cotidianamente (Knox \& Walker, 2001). Los resultados de la investigación nos alerta sobre el aprendizaje del consumidor, tanto por el consumo, como por la exposición a las estrategias publicitarias y nos muestra que de ambos obtiene información valiosa a la hora de tomar decisiones de consumo o compra.

Sería pertinente analizar los costos financieros de construir diferenciación de marca vía publicitaria o a través de los atributos de producto, de forma que se tengan elementos para tomar decisiones administrativas encaminadas a construir lealtades de marca con los menores costos y con una oferta de valor pertinente para el cliente.

\section{REFERENCIAS}

Caves, R., \& Williamson, P. (1985). What is product differentiation, Really? Journal of Industrial Economics 32 (2), 113-132.

Chen, G. R. (2014). How do advertised brands benefit from private labels? An application of rational expectations models. Applied Economics, 46 (24), 2891- 2902.

Colmenares, O., \& Saavedra, J. (2007). Aproximación teórica de la lealtad de marca: valoraciones y enfoques. Cuadernos de Gestión, 7 (2), 69-81.

Dinsmoor, J. E. (1995a). Stimulus control: Part I. The Behavior Analyst, 18, 51-68.

Foxall, G., Oliveira-Castro, J., \& Schrezenmaier, T. (2004). The behavioral economics of consumer brands choice: Patterns of reinforcement and utility maximization. Behavioral Process, 66, 235-260.

Froufe, M., \& Sierra, B. (1998). Condicionamiento clásico de las preferencias: implicaciones para la publicidad. Revista de Psicologia General y Aplicada, 51 (1), 85-104.

Hernández, R., Fernández-Collado, C., \& Baptista, P. (2006). Metodología de la investigación. México D.F.: Mc Graw Hill.

Inman, J., \& Zeelenberg, M. (2002). Regret in repeat purchase versus switching decisions: The attenuating role of decision justifiability. Journal of Consumer Research, 29, 116-128.

Janiszewski, C., \& Van Osselaer, S. (2000). A connectionist model of the brand quality association. Journal of Marketing Research, 37, 331-350.

Knox, S., \& Walker, D. (2001). Measuring and managing brand loyalty. Journal of Strategic Marketing, 99 (2), 111-128.

Martínez-Carrasco, L., Brugarolas, M., Del Campo, F., \& Martínez, 
A. (2006). Influence of purchase place and consumption frequency over quality wine preferences. Food Quality and Preference 17, 315-327.

Ortíz, J. (2013). Principios de Estadística Aplicada. Bogotá, D.C.: Ediciones de la U.

Porter, M. (2004). Estrategia Competitiva. México, D.F.: CECSA.

Romaniuk, J., Sharp, B., \& Ehrenberg, A. (2007). Evidence concerning the importance of perceived brand differentiation. Australasian Marketing Journal, 15 (2), 42-54.

Rundle-Thiele, S. (2005). Loyalty: An empirical exploration of theoretical structure in two service markets. Disertación doctoral. Australia: University ot South Australia .

Samuelson, W., \& Zeckhauser, R. (1988). Status quo bias in decision making. Journal of Risk and Uncertainty, 1, 7-57.

Schweitzer, M. (1994). Disnetangling status quo and omission effecs: An experimental analysis.OrganizationalBehaviorand Human Decision Process, 58, 457-476.

Trout, J., \& Rivkin, S. (2000). Differentiate or die: survival in our era of killer competition. New York: John Wiley \& Sons, Inc.

Tversky, A., \& Kahneman, D. (1974). Judgement under uncertainty: Heuristic and biases. Science, 185 (4157), 1124-1131. Van Osselaer, S., \& Janiszewski, C. (2001). Two ways of learning brand associations. The Journal of Consumer Research, 28 (2), 202-223.

Vandenbosch, M., \& Weinberg, C. (1995). Product and price competition in a two-dimensional vertical differentiation model. Marketing Science, 14(2) , 224-249.

Wasserman, E., Yung, M., \& Cook, R. (2004). Variability discrimination in humans and animals: Implication for adaptative action. American Psychologist, 59 (9), 879-890. 